\title{
Zu den offenen Fragen des Board Primacy Konzeptes in der Theorie der Corporate Governance
}

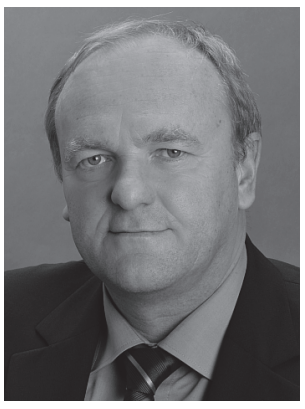

\section{Egon Franck}

Corporate Governance, Shareholder Primacy, Board Primacy, Treuhandpflichten, Business Judgement Rule

Corporate Governance, Shareholder Primacy, Board Primacy, Fiduciary Duties, Business Judgement Rule

Die Aktiengesellschaft ist ein Angebot aus dem „Regal“ des Rechts, bei dem spezifische Investoren ihre Ressourcen in einem Teamproduktionsprozess poolen, gegenseitig abrüsten und die residuale Kontrolle an unabhängige Direktoren übertragen. Deren weitreichende Diskretion wird im Wesentlichen durch die Verpflichtung begrenzt, als Treuhänder das Wohl der Firma und damit die Interessen aller spezifischen Investoren zu achten. Auch wenn die Wirksamkeit der Treuhandpflichten Fragen aufwirft, wird diese aber keineswegs durch ausgeprägte Sanktionen und Belohnungen für Direktoren erhöht.

The public corporation can be viewed as a pre-constructed legal arrangement, where specific investors agree to pool resources, disarm and cease residual control to independent directors. The discretion of the latter is only limited by the requirement to fulfill their fiduciary duties towards the corporation. While the effectiveness of fiduciary duties remains an issue of dispute, high-powered incentives and sanctions for corporate directors are clearly counter-productive.

\section{Einleitung}

Der ökonomische Mainstream propagiert bekanntlich die Shareholder Primacy in der Corporate Governance. Folgt man jedoch der innerökonomisch vorgebrachten Kritik, lässt sich die Vorstellung einer Shareholder Primacy nachvollziehbar und so lange aufweichen, bis der übrig gebliebene Rest ganz im Einklang zu der institutionellen Realität der Board Primacy in der Corporate Governance steht.

Obzwar die Aufweichbewegung logisch erscheint, konnte sie den Mainstream bislang nicht ablösen. Das hängt auch damit zusammen, dass der übrig gebliebene Rest nur ein unterbestimmtes Board Primacy Konzept hervorbringt, in dem mehr darüber ausgesagt wird, was Board Primacy „nicht ist“, als was sie „ist“. Eine solche Schattenvariante der Board Primacy übt gegenüber dem Alternativkonzept der Shareholder Primacy, das mittlerweile zu einem ganzen Arsenal von Überwachungs- und Anreizinstrumenten im Kontext des Prinzipal-Agenten-Problems zwischen Aktionären und Direktoren ausdifferenziert wurde, wenig Attraktivität aus. 
Im Folgenden interessieren vor allem folgende Fragen: Warum führt die innerökonomische Kritik am Mainstream zu einem unterbestimmten Board Primacy Konzept? Lässt bzw. wie lässt sich das beheben? Was folgt daraus für die Theorie der Corporate Governance?

\section{Subjektive Skizze der ökonomischen Theorie der Corporate Governance}

In einem früheren Beitrag ${ }^{1}$ wurde den ökonomischen Herleitungen der Shareholder Primacy und der Board Primacy in der Corporate Governance ausführlich Raum gewidmet. Im Folgenden wird diese Analyse nur kurz angedeutet.

Aus der ökonomischen Firmentheorie herkommend, wie sie am klarsten Jensen/Meckling (1976) und Fama/Jensen (1983a, 1983b) formuliert haben, gelangt man zur Konzeption des Aktionärs als des eigentlichen Halters der Residualansprüche in der so genannten Modern Corporation. ${ }^{2}$ Damit entsteht automatisch eine problematische Situation: Die einzige schädigbare Partei - der Aktionär, der keinen Vertrag mit fixierten Ansprüchen hat fällt die Entscheidungen über den Ressourceneinsatz in der Firma - also die Entscheidungen mit Schädigungspotential - nicht mehr selbst. Eine dritte Partei, die Direktoren, fällen diese stattdessen.

Wem sollten die Direktoren verantwortlich sein? Das ist offensichtlich: Den eigentlichen Haltern von Residualansprüchen, den Aktionären, weil nur sie zu Opfern werden können. Von allen Beteiligten haben nur sie sich auf eine unbestimmte „Restentschädigung“ eingelassen, die von den Direktoren manipulierbar ist. Wird unterstellt, dass Direktoren eigene Interessen haben, die sie aufgrund von Informationsasymmetrien auch auf Kosten der Aktionäre verfolgen könnten, ist man im Mainstream angekommen. Die Shareholder Primacy folgt zwingend aus dieser Theorie. Corporate Governance kann nur davon handeln, wie den ausgelieferten Aktionären, den Prinzipalen, über diverse Überwachungs- und Anreizsysteme eine Art „verlängerter Arm“ gebaut werden kann, damit sie den Konsum des Shareholder-Value durch ihre Agenten, die Direktoren, wirksam verhindern können.

Die Annahme, wonach der Aktionär der eigentliche Halter der Residualansprüche sei, ist schon frühzeitig innerhalb der Ökonomie selbst kritisiert worden. ${ }^{3}$ Verschiedene Autoren haben betont, dass z.B. auch Mitarbeiter illiquide, firmenspezifische Investitionen in ihr Humankapital tätigen, die sie im gleichen Sinne zu Risikoträgern werden lassen, wie es

1 Vgl. zu den Ausführungen in diesem Abschnitt 2 ausführlich Franck (2011, 204 ff.).

2 Wie man diese Position besonders überzeugend ableitet, hat Kürsten (2013) eindrucksvoll dargelegt. Es genügt im Wesentlichen die Annahme rationaler Erwartungen und rationalen Verhaltens der Stakeholder, damit ein nicht-monistisches Shareholder Value-Prinzip entsteht, welches das Aktionärsinteresse automatisch auf den Unternehmenswert als Ganzes lenkt. Die Publizität der Managerentlohnungsstruktur (nicht die Lohnhöhe!) wird in diesem Kontext zum Kernthema, denn sie ermöglicht es allen annahmegemäss rationalen Stakeholdern, die Manager-Entscheidungen zu antizipieren und den Shareholdern „in Rechnung“ zu stellen. Als Folge orientieren sich diese am Unternehmensgesamtwert, denn sonst müssten sie die „Zeche“ in Gestalt von Wohlfahrtsverlusten selbst zahlen. Insgesamt sind rationale Shareholder, die alle Hedging-Strategien rationaler Stakeholder antizipieren, daher die einzigen Halter von Residualansprüchen.

3 Diese Kritik unterstellt offenbar, dass Stakeholder Managerentscheidungen nicht von vornherein antizipieren und in ihre Vertragsverhandlungen einbauen können. Sonst würde die von Kürsten (2013) formulierte Lösung gelten, aus der die Orientierung am Unternehmensgesamtwert als aufgeklärtes Shareholder-Value-Prinzip folgt. 
die Aktionäre sind. ${ }^{4}$ Dies zeigt sich z.B., wenn Unternehmen unter finanziellen Druck geraten und Mitarbeiter ihre Entschädigungsansprüche nach unten korrigieren müssen. Soweit der Lohnanspruch der Mitarbeiter ihre unternehmensspezifischen Fähigkeiten betrifft, bezieht er sich also offenbar ebenfalls auf das Residuum, das nach Abzug aller besser durchsetzbaren gesetzlichen und vertraglichen Verpflichtungen im Unternehmen anfällt. Da sowohl die Dividenden für die Aktionäre wie auch die Löhne für spezifisches Humankapital der Mitarbeiter aus dem „gleichen Topf“ bezahlt werden, konkurrieren Gehaltssteigerungen für die Mitarbeiter mit Gewinnsteigerungen für die Aktionäre.

Im Kontext dieses unentrinnbaren Verteilungskonfliktes wird daher eine Art „Rüstungsbalance“ zwischen Aktionären und spezifisch investierenden Mitarbeitern zur „Zauberformel“. Würde man z.B. einseitig die Aktionärsrechte - wie etwa in der Schweiz im Rahmen der so genannten Abzockerinitiative (vgl. Initiativkomitee 2011) gefordert - stärken, könnten Mitarbeiter als Reaktion weniger bereit sein, unternehmensspezifische Fähigkeiten aufzubauen. Sie müssten systematisch befürchten, nachträglich von erstarkten Shareholdern um ihre Rückflüsse betrogen zu werden. Wenn also eine „Bewaffnung“ der Aktionäre durch mehr Mitsprachrechte erfolgen soll, dann nur Hand in Hand mit einer ebensolchen der Mitarbeiter. ${ }^{5}$ Würde man aber eine solche „symmetrische Aufrüstungslösung“ per Gesetz durchsetzen, dann käme es zur Umwandlung der heute in den meisten Ländern unabhängigen Boards in Interessenvertreter-Gremien, die aus Delegierten statt aus Direktoren bestünden.

Diese „Rüstungsbalance zwischen den spezifischen Investoren auf hohem Niveau“ trifft aber ebenfalls auf Einwände. Ein mit Interessenvertretern besetztes Führungsgremium der Aktiengesellschaft verwandelt sich nämlich immer dann in eine Arena der politischen Rentensuche, wenn die Interessendivergenzen der vertretenen Stakeholder erheblich sind (vgl. Anabtawi 2006; Bainbridge 2006; Stout 2007). Neben die Interessendivergenz zwischen Aktionären und Mitarbeitern treten solche innerhalb der Shareholder, wie z.B. Anabtawi (2006) unter dem vielsagenden Begriff Shareholder Sharking ausführlich untersucht hat. Darüber hinaus sind - wie etwa Hansmann (1993, 593) beschreibt - Mitarbeiterinteressen sprichwörtlich schwer aggregierbar zu einem allgemein akzeptierten Standard, den die Beteiligten selbst oder auch Dritte, wie z.B. Gerichte, in Konfliktsituationen zur Problemlösung anwenden könnten. Vor diesem Hintergrund droht ein mit Interessenvertretern besetztes Führungsgremium der Aktiengesellschaft zur Arena der Rentensuche mit eskalierenden Politikkosten zu werden.

Alternativ kann daher auch an eine „Rüstungsbalance zwischen spezifischen Investoren auf tiefem Niveau“ gedacht werden. Und plötzlich taucht die Idee der Board Primacy am Horizont einer Theorie der Corporate Governance auf. Board Primacy kann nämlich als eine Art „Abrüstungsgleichgewicht“ der spezifischen Investoren angesehen werden. Sie impliziert nämlich gerade keine Shareholder Primacy und auch keine Employee Primacy und daher nichts anderes als eine „Selbstentwaffnung“ der spezifischen Investoren (vgl. Rajan/ Zingales 1998; Blair/Stout 1999, 2001; Stout 2003a). Aktionäre und Mitarbeiter bringen dann ihre Beiträge gezielt und bewusst in solchen Unternehmen ein, in denen sie wegen der im Vorfeld festgelegten Board Primacy die „Macht des Eigentums“ nicht mehr gegenein-

4 Vgl. hierzu vor allem Blair (1995, 1996, 1999), aber auch z.B. Furubotn (1988), Hansmann (1993), Blair/Stout (1999), Stout (2007), usw.

5 Vgl. allgemein zur Exit-Voice-Problematik Hirschman (1970); Vgl. für eine breitere Perspektive der „Employee governance“ Brink (2011, 58), Osterloh/Frey (2006). 
ander einsetzen können. Der eigenen Schutzlosigkeit wird durch die Nichtangriffsfähigkeit der anderen spezifischen Investoren die Brisanz genommen. Board Primacy schafft Anreize für unternehmensspezifische Investoren, gerade weil sie deren politische Rentensuche unterbindet. ${ }^{6}$

So überzeugend das aus der Idee einer „Rüstungsbalance auf tiefem Niveau“ abgeleitete Konzept der Board Primacy auf den ersten Blick auch erscheinen mag, so hilflos hinterlässt es einen bei näherem Hinsehen. Genau genommen macht das „Abrüstungsgleichgewicht“ nur deutlich, was Direktoren nicht sein können, nämlich Agenten der Aktionäre oder einer anderen Gruppe spezifischer Investoren. Sie führt aber nicht aus, was Direktoren sein sollten und woran „gute“ Direktoren gemessen werden können. Anders gesagt: Der Auftrag des Board kann nicht allein darin bestehen, kein Auftragnehmer der Aktionäre oder einer anderen Stakeholdergruppe zu sein.

Die bisher skizzierten ökonomischen Überlegungen lassen daher Fragen offen, zwischen dem, was Board Primacy „nicht ist“ - nämlich keine Shareholder Primacy und auch keine irgendwie geartete Stakeholder Primacy - und dem, was Board Primacy „ist“. Die folgenden Überlegungen stossen in dieses offene Feld, gestützt auf anderen Literaturgrundlagen, vor.

\section{Die Abstraktion von der Unternehmung als Akteur}

Wenn die Direktoren nicht Auftragnehmer der Aktionäre, Mitarbeiter oder anderer Stakeholder sind, was sind sie dann?

Es fällt deswegen so schwer, einen Bezugspunkt für die Beschreibung der Rolle der Direktoren $\mathrm{zu}$ finden, weil die ökonomischen Governance-Theorien mit einer weiteren folgenschweren Setzung operieren, auf die Walgenbach (2011, 313) zu Recht hinweist: Sie blenden die Unternehmung selbst als handelnden Akteur vollständig aus der Betrachtung aus. Diesen Kunstgriff leistet die auf Jensen/Meckling (1976) zurückgehende Sicht der Firma als eines Knotenpunkts von Verträgen:

"It is important to recognize that most organizations are simply legal fictions which serve as a nexus for a set of contracting relationships among individuals." (Jensen/ Meckling 1976, 310; Verweise im Zitat weggelassen)

Akzeptiert man diese Sichtweise, dann erscheint es nur folgerichtig, dass man durch die legale Firmenfiktion hindurchsehen muss, um den Blick ungestört auf die „eigentlichen“ Verträge zwischen den Individuen zu richten, die etwas „beitragen“. Ohne Umschweife kann dann in die unmittelbare Analyse dieser „tiefer liegenden“ Vertragsbeziehungen zwischen Aktionären, Direktoren und Mitarbeitern eingestiegen werden.

Tatsächlich ist die „nexus of treaties“-Sichtweise aber selbst nur eine Fiktion. So haben etwa die Mitarbeiter der Daimler AG - um ein Beispiel von Walgenbach (2011, 313-314) zu nennen - weder einen Vertrag mit Herrn Dr. Zetsche noch einen Vertrag mit irgendeinem Aktionär, sondern einen Vertrag mit der Daimler AG, die gemäss $\mathbb{S} 1$ des deutschen Aktiengesetzes ein Akteur mit eigener Rechtspersönlichkeit ist. Die in der Idee der Firma als legale Fiktion verfangenen ökonomischen Ansätze konzentrieren sich also nur auf ver-

6 Oder wie Stout $(2007,797)$ ausführt: „Board governance, as a result, attracts specific investors by offering...the promise of ,business continuity““. 
meintliche Verträge zwischen Individuen, die nirgendwo auf einem Stück Papier aufgeschrieben sind.

Das Ignorieren der Realität in einem Denkwerkzeug ist aber prinzipiell legitim. Schwerwiegend an diesem blinden Fleck der „nexus of treaties“-Sichtweise ist jedoch, dass die darauf aufbauenden Denkwerkzeuge weitgehend unbrauchbar sind, um die Rolle eines Board of Directors und damit einen essentiellen Aspekt der Corporate Governance angemessen zu untersuchen. In der juristischen Literatur - stellvertretend seien hier die Beiträge von Blair/Stout (1999) und Bainbridge (2002a,b,c) genannt - wie auch in der Managementliteratur - hier sei exemplarisch auf Lan/Heracleous (2010) und Walgenbach (2010, 2011) verwiesen - wurde das Konzept der Board Primacy im Kontext von GovernanceTheorien weiterentwickelt, in denen die Unternehmung als Akteur inkludiert ist.

\section{Board Primacy als „besonderes Auftragsverhältnis“ Firma-Direktoren}

Tatsächlich entsteht durch Inkorporierung mit der Firma selbst eine eigenständige juristische Person:

„When a corporation is formed, a new legal entity is born. As a matter of law, this entity, and not any of its participants, owns the assets used in corporate production, as well as any surplus produced by the enterprise." (Blair/Stout 2001, 423)

Der neu entstandene Akteur Unternehmung ist also Eigentümer der von den verschiedenen Inputlieferanten eingebrachten Ressourcen. Dies gilt insbesondere auch für das bereitgestellte Kapital der Aktionäre:

„Once shareholders subscribe to shares in the corporation, payment made in consideration for the shares is considered property of the corporation, and the shareholders are not free to withdraw the sum invested except for payments through dividends, selling their shares, and other permitted means. Shareholders own the shares, not the corporation itself, which is an autonomous legal entity. As Fama noted, Ownership of capital should not be confused with ownership of the firm'. "(Lan/Heracleous 2010, 301; Literaturverweise im Zitat weggelassen)

Der neu entstandene Akteur Firma hält nicht nur das Eigentum an allen eingebrachten Ressourcen, sondern geht Rechtsgeschäfte im eigenen Namen ein, ohne die einzelnen Aktionäre oder Mitarbeiter dadurch zu verpflichten. Folgerichtig haftet die Firma auch als juristische Person für die eigenen Verbindlichkeiten. ${ }^{7}$

Eigentum an Ressourcen, das Eingehen von Rechtsgeschäften sowie Haftung sind klare Belege einer ökonomischen „Existenz“ des Akteurs Firma. Mit Bezug auf diesen Akteur ist nun die Rolle des Boards als ein besonderes Auftragsverhältnis definiert.

Die Firma selbst als juristische Person ist der „Auftraggeber“ des Boards. Weder die einschlägigen Rechtsvorschriften noch die Rechtsprechung lassen Zweifel daran aufkommen. Die Direktoren haben den Auftrag, Entscheidungen über den Einsatz der in das Eigentum der Firma übergegangenen Ressourcen zum „Wohle der Firma“ zu treffen. Dies kann z.B. in $\mathbb{S} 93$ (1) des deutschen Aktiengesetzes nachgelesen werden, wo vom „Wohle der Gesellschaft“ die Rede ist, oder in Section 172 (1) des U.K. Companies Act, wo vom „success of the company for the benefit of its members as a whole“ gesprochen wird, oder in Artikel

7 Vgl. dazu ebenfalls Lan/Heracleous (2010, 301) mit Verweisen auf Ferran (1999) und andere. 
717, Absatz 1 des Schweizerischen Obligationenrechts, wo ausgeführt wird, dass Mitglieder des Verwaltungsrats „...die Interessen der Gesellschaft in guten Treuen wahren“.

Direktoren sind also Auftragnehmer des durch Inkorporierung neu entstandenen Akteurs Firma (vgl. hierzu Lan/Heracleous 2010, 301). Ihre Verpflichtung auf das Wohl der Fima ermöglicht ihnen sogar, gezielt gegen die Interessen einzelner Gruppen, wie etwa der Aktionäre, zu entscheiden. Auch daran lassen weder Rechtsprechung noch Rechtslage irgendwelche Zweifel. Lan/Heracleous (2010, 300-301), tragen vielfältige Evidenz zusammen, dass Gerichte Entscheidungen des Boards „auf Kosten“ der Aktionäre regelmässig stützen, wenn diese dem langfristigen Wohl der Firma durch einen verbesserten Schutz der Mitarbeiter, der Gläubiger usw. zu Gute kommen.

In der juristischen Literatur wird die Besonderheit des Auftragsverhältnisses zwischen Firma und Direktoren, das als „sui generis“ gilt (vgl. z.B. Bainbridge 2002c; Lan/Heracleous 2010, 301), also nicht in die übliche Formtypologie der Auftragsverhältnisse passt, sehr betont. Per Gesetz gibt die Firma nur einen dauerhaft gültigen und relativ abstrakten Auftrag an die Direktoren, das Wohl der Firma zu verfolgen. Diese erhalten die dafür notwendige Macht ebenfalls durch das Gesetz und nicht etwa von den Aktionären oder sonst woher. Immer wieder wird deswegen in der Literatur herausgestellt, dass die Macht der Direktoren „original and undelegated“ (vgl. z.B. Bainbridge 2006, 1756) sei. Lan/Heracleous (2010) zitieren in diesem Kontext Judge Walter am Obersten Gericht von Oregon:

"It...is true that corporate directors are not in any strict sense agents of the stockholders, that their powers are conferred upon them by law, and in a very important sense are original and undelegated, that they have an obligation to act for the corporation, that they are to a very large extent free from stockholder control, and that no agreement or by-law which deprives them of their power to act for and in the best interest of the corporation is valid. "(zitiert nach Lan/Heracleous 2010, 302)

Gerade weil die Firma eine juristische Person ist, die einen einmaligen und generellen Auftrag erteilt, stattet das Gesetz die Direktoren automatisch mit einer grossen diskretionären Machtbasis aus.

Als einzige „flankierende Massnahme“ zur Ausstattung mit einer derartigen Machtfülle werden die Direktoren gleichzeitig in eine treuhänderische Pflicht (Fiduciary Duty) genommen (vgl. u.a. Blair/Stout 2001; Stout 2002; Bainbridge 2002a). Dahinter steht ein Rechtskonzept, das auf den Leitideen der Sorgfalt, Loyalität, Aufrichtigkeit und des guten Glaubens beruht. Direktoren sind demnach ganz spezielle Auftragnehmer der Firma, nämlich deren Treuhänder. Sie verletzen ihre Treuhandpflicht, wenn sie Bilanzbetrug begehen oder Ressourcen der Firma „veruntreuen“. Von einem Treuhänder wird erwartet, dass er stets im Interesse seines Auftraggebers handelt, auch wenn ihn dieser nicht überwacht oder ihn prinzipiell gar nicht überwachen kann (Lan/Heracleous 2010, 302).

Vergleicht man das Treuhandverhältnis mit dem ökonomischen Prinzipal-Agenten-Verhältnis, so besteht ein wesentlicher Unterschied in den Erwartungen: Das ökonomische Prinzipal-Agenten-Verhältnis unterstellt einen eigennützigen Agenten, der durch Kontrollen, Anreize und Sanktionen erst dazu bewegt werden muss, die Interessen seines Prinzipals zu verfolgen. Das Treuhandverhältnis erwartet ganz im Gegenteil dazu einen Auftragnehmer, der von sich aus loyal handelt. 


\section{Die offene Frage: Wie wirksam ist das Konzept der „fiduciary duties“?}

Das Treuhänderrecht exponiert Direktoren zumindest theoretisch erheblichen Sanktionen. So haften Direktoren nach amerikanischem Recht grundsätzlich für die gesamten finanziellen Schäden aus einer fahrlässigen Entscheidung (Jones 2006, 113). Die drakonische Höhe der möglichen Sanktionen wird jedoch in der Praxis durch ihre systematische Nichtanwendung so verwässert, dass Kritiker von einer „,no liablity“ rule and the absence of accountability“ (Jones 2006, 117) sprechen. Berühmt ist das Zitat von Stout (2003b, 7) über die Wirksamkeit der Sanktionierung von Verletzungen der Sorgfaltspflicht. Demnach ist es für Direktoren „....more likely to be attacked by killer bees than she is to have to ever pay damages for the breach of the duty of care (a.a.O.) ".

Das Hauptvehikel zur Isolierung der Direktoren gegen die Sanktionen des Treuhänderrechts ${ }^{8}$ bietet die so genannte Business Judgement Rule, die nicht nur in den USA sondern z.B. auch in der Schweiz und in Deutschland ${ }^{9}$ zur Anwendung kommt. Wenn ein Direktor bei einer unternehmerischen Entscheidung vernünftigerweise annehmen durfte, auf der Grundlage angemessener Information zum Wohle der Firma zu handeln, kann ihm gemäss dieser Regel keine Pflichtverletzung vorgeworfen werden. Gerichte beschränken sich damit auf die Betrachtung des Prozesses, durch den eine Entscheidung des Boards zu Stande kam, und nicht auf die Qualität derselben (vgl. Jones 2006, 111). Der Nachweis einer Pflichtverletzung ist aus verschiedenen strukturellen Gründen, wie z.B. dem eines unterlegenen Zugangs der Aktionäre zu relevanten Informationen, nur äusserst schwer zu erbringen, so dass die derivative Aktionärsklage nur bei offensichtlichen und sehr krassen Verstössen gegen die Treuhandpflichten Wirkung entfalten kann. Wie lässt sich diese Sachlage interpretieren?

\subsection{Board Primacy zu Ende gedacht: „No liability“ als Preis, den die Firma für die} Aussetzung anderer „Rechenschaften“ bezahlt

Die Beschränkung der Rechenschaftspflicht der Direktoren gegenüber den Aktionären und anderen Stakeholdern ist sinnvoll. Ohne sie würden Direktoren letztlich doch zu Agenten von „Souffleuren“ aus dem Hintergrund degradiert. Die Vorteile der „Abrüstung spezifischer Investoren auf tiefem Niveau" verlangen nach einer konsequenten Umsetzung der Board Primacy in der Corporate Governance. Es verwundert daher auch nicht weiter, dass der Schutz der Direktoren zur Wahrung ihrer Autorität das erklärte Ziel der Business Judgement Rule ist. Dazu stellt z.B. das Oberste Gericht von Delaware - einem der beliebtesten Inkorporationsstaaten der USA - klar:

„The business judgment rule exists to protect and promote the full and free exercise of the managerial power granted to the Delaware directors." (Zitiert nach Bainbridge 2006, 1747)

Der unvermeidbare Nebeneffekt dieser systematischen Begrenzung der Rechenschaftsbegehren der Aktionäre und anderer Stakeholder liegt auf der Hand. Sie geht mit einer gleichzeitigen Behinderung der Rechenschaftsablage der Direktoren gegenüber der Firma einher. Die Firma als juristische Person ist nämlich im Normalfall auf ihre Direktoren an-

8 Für weitere Mechanismen, die den Effekt einer Isolierung der Direktoren gegen Sanktionen vervollständigen, siehe ausführlich Jones (2006, $113 \mathrm{ff}$.).

9 Wo sie in $\$ 93$ Abs. 1 Satz 2 AktG kodifiziert wurde. 
gewiesen, um ihre Rechte gegenüber Dritten vor Gericht einzuklagen. Ganz offensichtlich ist dieser "Normalweg“ nicht begehbar, wenn die Direktoren selbst - wie im Falle der Verletzung der Treuhänderpflichten - in den beklagenswerten Rechtsbruch involviert sind (vgl. Jones 2006, 113). Andere Akteure müssten in diesem Fall zu Agenten der Firma gemacht werden können, damit sie stellvertretend die Rechte der Firma gegen die „untreuen“ Direktoren durchsetzen. Indem die Business Judgement Rule sehr hohe Hürden an die Zulassung einer derivativen Aktionärsklage stellt, begrenzt sie automatisch auch die Möglichkeiten der Firma selbst, über gerichtlich eingesetzte Stellvertreter Rechenschaft von ihren Direktoren zu verlangen.

Einerseits schützt also die Business Judgement Rule alle anderen Stakeholder davor, dass die Direktoren über häufige derivative Aktionärsklagen dann doch in eine direkte Rechenschaftspflicht gegenüber den klagenden Aktionären genommen und zu deren Agenten degradiert werden. Andererseits behindert sie aber gleichzeitig die Firma, Rechenschaft von ihren Direktoren zu verlangen, denn im Falle „untreuer“ Direktoren wäre die Firma gerade auf andere Akteure angewiesen, die in ihrem Namen auftreten könnten.

Die Ausgangslage erscheint daher paradox. Auf der einen Seite formuliert das Treuhänderrecht einen hohen Anspruch an die Direktoren, der sich in potentiell hohen Sanktionen für den Fall der Untreue niederschlägt. Auf der anderen Seite sind diese Sanktionen so gut wie undurchsetzbar. Übersetzt in ein klassisches ökonomisches Kalkül stellt sich die Entscheidungssituation der Direktoren wie folgt dar. Diese kalkulieren stets die erwarteten persönlichen Kosten gegen den erwarteten persönlichen Nutzen eines Bruchs der Treuhandpflichten. In die Berechnung der Kosten gehen dabei die Wahrscheinlichkeit der Bestrafung eines Regelbruchs sowie die Höhe der Strafe ein. Da das „no liablity“-Regime die Wahrscheinlichkeit der Bestrafung auf Null senkt, sollte die Pflichtverletzung unter Direktoren zur Regel werden, sobald Untreue irgendwelche persönlichen Erträge abwirft.

\subsection{Das Treuhänderrecht als ,sheep dog“}

Man kann sich aber auch fragen, ob das skizzierte ökonomische Kalkül eine gute Beschreibung der Regelbefolgung und damit der Rolle des Rechts für die Steuerung menschlichen Verhaltens abgibt. Eine Vielfalt von psychologisch und soziologisch fundierten Arbeiten, die man grob unter dem Begriff des „law and norms approach“ in der Rechtswissenschaft zusammenfassen kann, propagiert eine andere Sicht: ${ }^{10}$ Sanktionen haben demnach nicht die Rolle, das persönliche Erwartungsnutzenkalkül der Akteure unmittelbar so zu verändern, dass sich die Einhaltung der Gesetze individuell lohnt. Dies wäre oft nur zu prohibitiven Kosten möglich. Eine viel wichtigere Rolle spielt die innere Einstellung zu dem in einem Gesetz ausgedrückten Verhalten:

„Widespread voluntary compliance with law is essential to maintaining social order...As one example, our income tax regime relies primarily on self-reporting by taxpayers. These self-reporting obligations are supported by a monitoring system and a penalty regime for those who fail to fulfill their obligations. Despite these mechanisms, the actual risk of being detected for cheating remains low. Thus, the fear of being caught cannot explain why people pay taxes. Instead, theorists speculate that people pay taxes because they believe they should and because they believe their fellow citizens

10 Vgl. hierzu und zum Folgenden ausführlich Jones (2006, 121ff.), der eine umfassende und gleichzeitig sehr kritische Würdigung dieses Ansatzes liefert. 
are also doing their part. If either of these beliefs were seriously undermined, compliance rates would likely falter. " (Jones 2006, 147; Literaturverweise im Zitat weggelassen)

Entscheidend ist daher vor allem die indirekte Wirkung von Gesetzen, die im besten Fall zur Verstärkung vorteilhafter und zur Schwächung nachteiliger sozialer Normen beitragen. „Gute“ Gesetze haben vor allem eine expressive Funktion, die einen Wandel in der sozialen Bedeutung und Erwünschtheit bestimmter Verhaltensweisen nach sich ziehen kann. ${ }^{11}$ Beispielsweise haben Gesetzesänderungen wie die Gurtpflicht beim Autofahren oder das Rauchverbot in Restaurants und öffentlichen Gebäuden zu einer sozialen Neubewertung des Fahrens ohne Gurt und des Rauchens in der Öffentlichkeit geführt. ${ }^{12}$ Sind „neue“ soziale Normen erst einmal etabliert, kommt es automatisch in einem Prozess der Selbst-Governance zu deren Durchsetzung. Grundsätzlich werden dabei zwei Durchsetzungswege unterschieden, die sich auch gegenseitig ergänzen und verstärken können (vgl. Jones 2006, 126).

Einerseits entfaltet sich die disziplinierende Wirkung der Normen unmittelbar im sozialen Zusammengang. Wie ein einzelner Raucher im Lichthof der Universität Zürich heute anders als vor 10 Jahren breite öffentliche Empörung fürchten muss, halten nach dieser Sicht der Dinge Direktoren zur sozialen Norm avancierte Treuhandpflichten ein, um Verlegenheit, Scham oder Ausgrenzung in der Geschäftswelt zu vermeiden. ${ }^{13}$ In erster Linie funktioniert die Durchsetzung sozialer Normen aber über internalisierte Präferenzen. ${ }^{14}$ So wie eine „Nichtrauchereinstellung“ eine günstige innere Überzeugung in einer Welt mit Rauchverboten sein kann, könnte auch die Verinnerlichung der Treuhandpflichten unter fairen Geschäftspartnern zur Vermeidung kognitiver Dissonanzen beitragen.

Indem sich der Staat über die Ausgestaltung des Rechts dem „Normenmanagement“ zuwendet, anstatt individuelle Erwartungsnutzenkalküle direkt über Sanktionen beeinflussen zu wollen, kann er in dieser Sicht der Dinge unerwünschtes Verhalten effektiver und billiger bekämpfen (Sunstein 1996, 908; Jones 2006, 124). In diesem Sinne gilt:

„...the law plays the role of a sheep dog, but does not intervene more than necessary." (Rock/Wachter 2001, 1662)

Aber wie viel ist wirklich „necessary“? Niemand bezweifelt, dass „geeignete“ soziale Normen, die von vielen Direktoren internalisiert werden, einen Beitrag zur Durchsetzung der Fiduciary Duties leisten. Aber wie kann das Treuhänderrecht dies begünstigen?

5.3 Die offene Frage: Wie kalibriert man wirksame Ermahnungen statt (un)wirksamer Sanktionen für Direktoren?

Klassiker der psychologischen Literatur zeigen, dass zu starke Bestrafungen oder Belohnungen eine Internalisierung neuer Normen verhindern und nicht etwa begünstigen (vgl. Festinger/Carlsmith 1959; Aronson/Carlsmith 1963). Folgende Erklärung hat sich dafür eingebürgert (vgl. Jones 2006, 150): Wenn eine Person eine Handlung vollzieht, die im Widerspruch zu ihren bisherigen Überzeugungen steht, erzeugt dies eine kognitive Disso-

11 Vgl. Jones (2006, 123) mit dem Verweis auf Arbeiten von Lessig (1998), Posner (2000) und andere.

12 Für diese und andere Beispiele siehe Lessig (1995); Vgl. auch Jones (2006, 123-124).

13 Vgl. dazu ausführlich Rock/Wachter (2001); Vgl. auch hierzu Jones (2006, 124).

14 Jones (2006, 125 ff.) rechnet Blair/Stout (2001) zu Vertreterinnen dieser „internal motivations“ Richtung des „law and norms approach“. 
nanz. Diese kann grundsätzlich so verarbeitet werden, dass die Person ihre Einstellungen oder Überzeugungen revidiert. Je grösser nun aber die Belohnungen oder Bestrafungen werden, die zur Auslösung der kontraintuitiven Handlung angeboten bzw. angedroht werden, desto geringer fällt die in Experimenten gemessene Veränderung der Überzeugungen aus. Der Grund wird darin gesehen, dass eine starke externe Rechtfertigung für die kontraintuitive Handlung kaum oder wenig kognitive Dissonanz erzeugt. Eine Veränderung der ursprünglichen Einstellung und damit eine Verinnerlichung neuer Normen erweist sich gar nicht mehr als nötig. Der externe Zwang allein genügt, um die Handlung zu motivieren. Sobald dieser allerdings wegfällt, handelt die Person aus ihrer unveränderten Einstellung heraus genau wie früher. Im Ergebnis hat sich gezeigt:

„...mild threats have been found to be more effective than severe threats in inducing lasting behavioral change." (Jones 2006, 150)

Tatsächlich scheint das Treuhänderrecht als ein „mild threats“-Regime in diesem Sinne ausgestaltet zu sein. Auch wenn Gerichte zwar nur äusserst selten Klagen gegen Direktoren zulassen und diese dann am Ende so gut wie nie zu finanziellen Strafen verurteilen, kritisieren sie regelmässig zweifelhaftes Direktorenverhalten und sprechen Ermahnungen zur Einhaltung der Treuhänderpflichten aus. Jones (2006, 130) drückt dieses wie folgt aus:

„...corporate law and norms theorists argue that judges, through their legal opinions, telegraph to directors what society expects from them. These judicial opinions are presumed to carry moral force that help discourage fiduciary breaches even when courts ultimately decline to impose liability."

In dieser Sicht der Dinge leistet das „mild threats“-Treuhänderrecht bereits über solche Ermahnungen einen Beitrag zur Internalisierung der Treuhändernormen durch die Direktoren. Darüber, wie die „mild threats“ speziell im Kontext der Corporate Governance genau kalibriert sein müssen, um wirksam zu sein, gibt es einige Spekulationen, aber kaum erhärtete Fakten. ${ }^{15}$

Wenn allerdings die grundsätzlichen Überlegungen so zutreffen, dann gilt es aufzupassen, dass diese „mild threats“ Situation, die das Recht vorstrukturiert, nicht auf Unternehmensebene systematisch unterlaufen wird. Das gesamte Amt des Direktors muss dann in das vom Recht vorgegebene „mild threats framework“ hineinpassen und eher auf die Internalisierung der Treuhänderpflichten statt auf die direkte Beeinflussung von Erwartungsnutzenkalkülen durch ausgeprägte Belohnungen oder Sanktionen ausgerichtet sein.

Dies bedeutet dann erstens, dass Direktoren nur symbolische Entschädigungen beziehen sollten (vgl. Jones 2006, 151). Hohe Löhne ermöglichen Handlungen der Direktoren auch gegen ihre inneren Überzeugungen. Dies ist kontraproduktiv in einem Umfeld, das ganz und gar auf die ausgeprägte „innere Überzeugungsarbeit“ von Direktoren setzt, um Treuhandpflichten zu internalisieren. Es bedeutet zweitens, dass die Entschädigungen der Direktoren fix und nicht etwa an die Erfüllung irgendwelcher Indikatoren gebunden sein sollten. Derartige externe „Leistungsanreize“ unterminieren nicht nur die angestrebte Normeninternalisierung. Sie stellen zudem die Unabhängigkeit der Direktoren in Frage, indem sie diese als Verbündete einzelner Gruppen spezifischer Investoren erscheinen lassen. Nur wer aber selbst kein „verlängerter Arm“ einer Interessengruppe ist, kann glaubwürdig das

15 Vgl. den Beitrag von Jones (2006) für einen Überblick über die wesentlichen Positionen und Fragen. 
System der „Entwaffnung“ der Interessengruppen symbolisieren (vgl. Blair/Stout 2001, 443; Franck 2011, 212).

Anders gesagt: Jeder Direktor, der hohe und erfolgsabhängige Entlohnungen etwa über Aktien oder Optionen akzeptiert, hat ein Rechtfertigungsproblem. Aufgrund starker externer Anreize misst er seine Entscheidungen nicht mehr an seinen inneren Überzeugungen. Die Wirkung der externen Belohnungen ersetzt die Auseinandersetzung mit kognitiven Dissonanzen. Es spielt dabei keine Rolle, ob Direktoren vom CEO „gekauft“ werden („managerial power“), oder ob sie eigenständig die Bereicherungsmöglichkeiten erkennen, die mit einer Entpflichtung aus ihrer Treuhänderrolle durch Stilisierung zum Agenten der Aktionäre einhergehen. Hohe und erfolgsabhängige Löhne signalisieren stets den Abschied von einem Regime verinnerlichter Treuhandpflichten.

\section{Schlussbetrachtung}

Die Verfassung der Aktiengesellschaft ist also ein Angebot aus dem Regal der durch die Rechtssetzung und -sprechung vorgefertigten Institutionen an spezifische Investoren, die bereit sind, Ressourcen in einem Teamproduktionsprozess zu poolen. Dieses Angebot aus dem Regal des Rechts lässt sich als ein kombinierter Abrüstungs- und Auslieferungspakt der spezifischen Investoren verstehen. Die Auslieferung erfolgt dabei an Direktoren, deren weitreichende Diskretion im Wesentlichen durch die Verpflichtung begrenzt wird, als Treuhänder der Firma das Allgemeinwohl und damit die Interessen aller spezifischen Investoren zu achten. Seine Qualität steht und fällt letztlich mit der Frage, wie wirksam das Konzept der Fiduciary Duties ist.

Diese Frage kann nicht abschliessend beantwortet werden. Dennoch erscheint ein positiver Ausblick angebracht. Die Sanktionsschwäche des Treuhänderrechts, die indirekt aus der Einschränkung der Rechenschaftspflichten der Direktoren gegenüber Aktionären und anderen Stakeholdern im Dienste einer konsequenten Board Primacy resultiert, ist wohl kein echtes Problem. Es stimmt zwar, dass der weitgehende Wegfall finanzieller Sanktionen das Erwartungsnutzenkalkül der Direktoren so beeinflusst, dass Untreue vorteilhaft erscheint, sobald die Pflichtverletzung irgendwelche individuellen Belohnungen generiert. Dies ist aber nur ein Problem, solange man die Frage nach wirksamen Sanktionen für Direktoren zur Kernfrage der Corporate Governance stilisiert.

Wenn Direktoren aber - wie andere Menschen auch - Normen deswegen einhalten, weil sie glauben, dass sie dies tun sollten (Jones 2006, 150), spielt dies keine so grosse Rolle. Im Gegenteil: Gerade der Wegfall substanzieller Sanktionen und Belohnungen nicht nur auf Gesetzes- sondern auch auf Firmenebene scheint eine Voraussetzung dafür zu sein, dass Treuhandpflichten durch Direktoren wirksam internalisiert werden können.

Natürlich bleibt die entscheidende Frage, wie man wirksame Ermahnungen kalibriert, um die Treuhandpflichten als soziale Norm unter Direktoren zu etablieren, im Detail noch weitgehend offen und Gegenstand zukünftiger Forschungen. Insofern kann man dem hier explizierten Board Primacy-Konzept immer noch Unvollständigkeit und vielleicht sogar Naivität vorwerfen, aber um mit Walgenbach (2010, 434) zu schliessen:

„Naivität sollte insofern in der Diskussion der Konzeptualisierungen der Unternehmung und der Diskussion der Instrumente rationaler Unternehmungssteuerung kein absolutes Argument sein, sondern sollte relativ gesehen werden. Die Fragen sind dann: 
Welche Theorie weist den höchsten Grad an Naivität auf? Und welche Theorie geht am naivsten mit der eigenen Naivität um und welche am wenigsten?"

\section{Literaturhinweise}

Anabtawi, I. (2006): Some Skepticism about Increasing Shareholder Power, in: UCLA Law Review, Vol. 53, unter http://cdn.law.ucla.edu/SiteCollectionDocuments/workshops \%20and\%20colloqui a/some \%20skepticism\%20about\%20increasing\%20shareholder\%20power.pdf (abgefragt am 10.2.2011).

Aronson, E./Carlsmith, J.M. (1963): Effect of the Severity of Threat on the Devaluation of Forbidden Behavior, in: The Journal of Abnormal and Social Psychology, Jg. 66, S. 584-588.

Bainbridge, S. (2002a): Why a board? Group Decision Making in Corporate Governance, in: Vanderbilt Law Review, Jg. 55, S. 1-55.

Bainbridge, S. (2002b): Director v. Shareholder Primacy in the Governance Debate, in: Transnational Lawyer, Jg. 16, S. 45-62.

Bainbridge, S. (2002c): Corporation Law and Economics, New York.

Bainbridge, S. (2006): Director Primacy and Shareholder Disempowerment, in: Harvard Law Review, Jg. 119, S. 1735-1758.

Blair, M.M. (1995): Ownership and Control, Washington D.C.

Blair, M.M. (1996): Wealth Creation and Wealth Sharing, Washington D.C.

Blair, M.M. (1999): Firm-Specific Human Capital and Theories of the Firm, Working Paper Nr. 167848, Georgetown University Law Center, unter http://papers.ssrn.com/sol3/Delivery.cfm/ SSRN_ID167848_code030912570.pdf?abstractid=167848\&mirid=1 (abgefragt am 10.2.2011).

Blair, M.M./Stout, L.A. (1999): A Team Production Theory of Corporate Law, in: Virginia Law Review, Jg. 85, S. 247-328.

Blair, M.M./Stout, L.A. (2001): Director Accountability and the Mediating Role of the Corporate Board, in: Washington University Law Quarterly, Jg. 79, S. 403-447.

Brink, A. (2011): Spezifische Investitionen als Legitimationsgrundlage für Stakeholderansprüche, in: Die Unternehmung, Jg. 65, S. 50-68.

Fama, E./Jensen M.C. (1983a): Separation of Ownership and Control, in: Journal of Law and Economics, Jg. 26, S. 301-325.

Fama, E./Jensen M.C. (1983b): Agency Problems and Residual Claims, in: Journal of Law and Economics, Jg. 26, S. 327-349.

Ferran, E. (1999): Company Law and Corporate Finance, Oxford.

Festinger, L./Carlsmith, J.M. (1959): Cognitive Consequences of Forced Compliance, in: The Journal of Abnormal and Social Psychology, Jg. 58, S. 203-210.

Franck, E. (2011): Ist es an der Zeit, die Aktionärsrechte zu stärken?, in: Die Unternehmung, Jg. 65, S. 201-214.

Furubotn, E.G. (1988): Codetermination and the Modern Theory of the Firm: A Property-Rights Analysis, in: Journal of Business, Jg. 61, S. 165-181.

Hansmann, H. (1993): Worker Participation and Corporate Governance, in: University of Toronto Law Journal, Jg. 43, S. 589-606.

Hirschman, A.O. (1970): Exit, Voice, and Loyalty, Cambridge.

Initiativkomitee (2011): Eidgenössische Volksinitiative "gegen die Abzockerei», unter http://www. volksinitiative-gegen-die-abzockerei.ch/ (abgefragt am 15.2.2012). 
Jensen, M.C. (2000): The Theory of the Firm, Cambridge.

Jensen, M.C./Meckling, W.H. (1976): Theory of the Firm: Managerial Behavior, Agency Costs, and Ownership Structure, in: Journal of Financial Economics, Jg. 3, S. 305-360.

Jones, R.M. (2006): Law, Norms, and the Breakdown of the Board: Promoting Accountability in Corporate Governance, in: Iowa Law Review, Jg. 92, S. 105-158.

Kürsten, W. (2013): 35 Jahre Jensen/Meckling und das Missverständnis um die (wahre) Zielfunktion der Aktionäre - Finanzierungstheoretisches Plädoyer zur Eignung der Principal-Agent-Theorie für die Lösung von Problemen der Corporate Governance, in: Die Unternehmung, Jg. 67, S. 8-22.

Lan, L.L./Heracleous, L. (2010): Rethinking Agency Theory: The View from Law, in: Academy of Management Review, Jg. 35, S. 294-314.

Lessig, L. (1998): The New Chicago School, in: The Journal of Legal Studies, Jg. 27, S. 661- 691.

Osterloh, M./Frey, B. (2006): Shareholders Should Welcome Workers as Directors, in: Journal of Management and Governance, Jg. 10, S. 325-345.

Posner, E. (2000): Law and Social Norms: The Case of Tax Compliance, in: Virginia Law Review, Jg. 86, S. 1781-1819.

Rajan, R.G./Zingales, L. (1998): Power in a Theory of the Firm, in: Quarterly Journal of Economics, Jg. 113, S. 387-432.

Rock, E.B./Wachter, M.L. (2001): Islands of Conscious Power: Law, Norms, and the Self-Governing Corporation, in: University of Pennsylvania Law Review, Jg. 149, S. 1619-1700.

Stout, L.A. (2002): Bad and not-so-bad Arguments for Shareholder Primacy, in: Southern California Law Review, Jg. 75, S. 1189-1209.

Stout, L.A. (2003a): The Shareholder as Ulysses: Some Empirical Evidence on Why Investors in Public Corporations Tolerate Board Governance, in: University of Pennsylvania Law Review, Jg. 152, S. 1-47.

Stout, L.A. (2003b): On the Proper Motives of Corporate Directors (Or, Why You Don't Want to Invite Homo Economicus to Join Your Board). Research Paper No. 03-8, UCLA School of Law, unter http://papers.ssrn.com/sol3/papers.cfm?abstract_id=389407 (abgefragt am 10.2.2011).

Stout, L.A. (2007): The Mythical Benefits of Shareholder Control, in: Virginia Law Review, Jg. 93, S. 789-809.

Sunstein, C.R. (1996): Social Norms and Social Roles, in: Columbia Law Review, Jg. 96, S. 903968.

Walgenbach, P. (2010): Das Ende der Organisationsgesellschaft und die Wiederentdeckung der Organisation, in: Die Betriebswirtschaft, Jg. 71, S. 419-438.

Walgenbach, P. (2011): Weg von der Agenturtheorie? Aber was dann?, in: Die Unternehmung, Jg. 65, S. 312-319.

Egon Franck, Dr., ist Professor für Strategische Unternehmensführung und -politik an der Universität Zürich.

Anschrift: Universität Zürich, Institut für Betriebswirtschaftslehre, Plattenstrasse 14, CH-8032 Zürich, Tel.: +41 (0)44/634-28-45, E-Mail: egon.franck@business.uzh.ch 